\title{
Pigeons' short-term memory for temporal and visual stimuli in delayed matching-to-sample
}

\author{
ROBIN L. BOWERS \\ Augustana College, Rock Island, Illinois \\ and \\ RALPH W. RICHARDS \\ Colorado State University, Fort Collins, Colorado
}

\begin{abstract}
In the present experiment, we compared directly pigeons' short-term memory of temporal and visual stimuli in a delayed matching-to-sample task. The sample stimuli consisted of red and green lights presented for 5 and $30 \mathrm{sec}$, followed by a retention interval and blue and yellow comparisons. For subjects in the visual group, duration was irrelevant and the color of the sample was the conditional cue. For animals in the temporal group, color was irrelevant and duration of the sample was the conditional stimulus. The results showed that acquisition of the matching task was faster and accuracy was higher in the visual than in the temporal group. More importantly, memory of either sample generally declined at a similar rate when the duration of the retention interval was increased and when the intertrial interval was reduced. Taken together, the results indicate that with 1-8-sec retention intervals, short-term memory for temporal stimuli is similar to that found with color-visual samples. The findings are discussed in terms of retrospective and prospective processing.
\end{abstract}

Over the past decade or so, the research examining pigeons' short-term memory (STM) has been extensive (e.g., Cook, 1980; Honig, 1978; Kendrick \& Rilling, 1984; Kendrick, Rilling, \& Denny, 1986; Roberts \& Grant, 1974, 1976; Roitblat, 1980; Roitblat, Bever, \& Terrace, 1984). Many of these studies employed the delayed matching-to-sample (DMTS) procedure. In DMTS, the subject is presented with a sample stimulus to which it responds. After the sample is terminated, a retention interval (RI) occurs, followed by a choice between two comparison stimuli, one of which matches or is identical to the previous sample. Responses to the matching comparison are reinforced and responses to the nonmatching comparison are not reinforced.

Recently, much experimental attention has focused on the content of STM. The basic question is whether subjects retain attributes of the sample during the RI (retrospective processing), or whether they retain an instructional code regarding the to-be-chosen comparison (prospective processing) (see Honig \& Thompson, 1982; Roitblat, 1980; Stonebraker, 1981; Wasserman, 1986, for further discussion). Kraemer and Roberts (1984) indirectly examined retrospective and prospective processing by

\footnotetext{
This paper represents a portion of the first author's dissertation presented to Colorado State University in partial fulfillment of the PhD requirement. Some of this work was presented at the 1986 meeting of the Rocky Mountain Psychological Association, Denver, CO. The authors would like to thank Marcia Spetch and the anonymous editors for their thoughtful comments, which improved the quality of the paper. Correspondence may be addressed to Robin L. Bowers, Department of Psychology, Augustana College, Rock Island, IL 61201.
}

training pigeons on a symbolic DMTS task in which visual or auditory samples were associated with visual comparisons. For some pigeons, the sample stimuli were 3000and $300-\mathrm{Hz}$ tones, and these were associated with yellow and blue, respectively, as the correct comparisons. For other pigeons, reinforcement for responding to the blue or yellow comparison depended on whether red or green was the sample. Kraemer and Roberts suggested that if the birds processed the samples retrospectively by retaining memories of visual and auditory sample attributes, then "we might expect to find quantitative and/or qualitative differences in retention of these stimuli" (p. 282). On the other hand, if the samples were processed prospectively by rapidly encoding the stimuli into response or choice instructions, Kraemer and Roberts suggested that there should be little difference in the slope of the forgetting curves following auditory and visual samples. Although several important findings emerged, their results showed that acquisition was more rapid and accuracy was higher in the visual than in the auditory group. Of greater interest, however, were the retention curves produced when accuracy was tested at the 0-, 0.5-, 1-, 2-, and 3sec RIs. The retention curves appeared to fall at roughly the same rate except during the first $0.5 \mathrm{sec}$, during which accuracy appeared to decline faster for the visual group. This difference, however, was largely due to the fact that accuracy was initially higher in the visual than in the auditory group. In a second experiment, the RI and intertrial interval (ITI) durations were varied together. In this condition, matching in both groups declined at approximately the same rate when the RI was increased, and increased similarly as the ITI was lengthened. Since the 
groups were affected similarly by the RI and ITI manipulations, Kraemer and Roberts concluded that similar STM processes occurred in each group. Specifically, they suggested that the auditory and visual subjects processed the samples prospectively, such that each type of sample activated a response instruction regarding the to-be-chosen comparison. To account for the superior performance shown by the visual group, Kraemer and Roberts suggested that the auditory samples were more difficult to encode, perhaps because the $300 / 3000-\mathrm{Hz}$ tones were less discriminable than the color samples (for further discussion, see Herman \& Forestell, 1985).

The purpose of the present study was to compare directly STM in a DMTS task with visual and temporal samples. More specifically, two groups of pigeons received sample stimuli (red and green lights presented for 30 and $5 \mathrm{sec}$ ), followed by an RI and the presentation of yellow and blue comparisons. For pigeons in the temporal group, responses to the yellow comparison were reinforced if the sample was $5 \mathrm{sec}$, regardless of the color of the sample. For animals in the visual group, the color of the sample was the relevant cue: responses to the yellow comparison were reinforced if the sample was green, and responses to blue were reinforced if the sample was red, irrespective of the sample duration. Following acquisition, performance was assessed when the RI was lengthened, and again when the RI and ITI durations were varied together.

This type of comparison is important because the retention curves obtained for event duration have not been compared directly with those obtained with other sense modalities. It is unclear, therefore, whether the underlying STM processes are similar or different for temporal and visual stimuli (cf. Cohen, Calisto, \& Lentz, 1981; Kraemer, Mazmanian, \& Roberts, 1985; Spetch \& Wilkie, 1982, 1983).

\section{METHOD}

\section{Subjects}

Fourteen experimentally naive, female White Carneaux pigeons served as subjects. All birds were maintained at approximately $80 \%-85 \%$ of their free-feeding weights. Water and health grit were available at all times in the home cages.

\section{Apparatus}

Two standard three-key operant conditioning chambers (internal dimensions: $34.5 \times 35.2 \times 35.5 \mathrm{~cm}$ ) were employed. Each chamber contained three Industrial Electronics Engineer projectors, one mounted behind each of the three keys. The two side keys were illuminated with yellow and blue lights and the center key was illuminated with white, red, and green lights. All display cells contained Sylvania 1820 bulbs. In addition, each chamber was illuminated by a houselight (Sylvania 1820 bulb) located $5.2 \mathrm{~cm}$ above the center key. Reinforcement was a 3-sec access to mixed grain presented in the food magazine located below the response keys. Presentation of the stimuli and the recording of key pecks were accomplished by separate Radio Shack TRS-80 microcomputers connected to each chamber via a Med Associates interface system.

\section{Procedure}

The 14 birds were randomly assigned to the visual and temporal groups. The subjects in each group were also randomly assigned to one of the two experimental chambers.

After being trained to eat from the food magazine, each bird was autoshaped to peck the center key illuminated by a white light. Each bird then received two sessions ( 40 reinforcements per session) of continuous reinforcement.

During initial DMTS training, all pigeons received 32 sessions (40 trials per session) in which a white light appeared on the center key, and the first response removed the light and produced the sample. The sample was a red or green light presented on the center key, and was terminated by the first response following 30 or $5 \mathrm{sec}$. Each sample type (red $30 \mathrm{sec}$, red $5 \mathrm{sec}$, green $5 \mathrm{sec}$, green $30 \mathrm{sec}$ ) was presented 10 times in each session in mixed order. A 0.5 -sec RI with darkened keys separated sample offset from the presentation of the blue and yellow comparisons that appeared on the side keys.

For the visual group, the color of the sample was the informative cue and the duration of the sample was irrelevant. These subjects were rewarded for choosing the yellow comparison after a green sample and for choosing blue after a red sample. For the temporal group, sample duration was the informative cue and color was irrelevant. Responses to the yellow comparison were reinforced after a 30-sec sample and responses to blue were reinforced after a 5-sec sample. If the incorrect comparison was chosen, the experimental chamber was darkened for $3 \mathrm{sec}$ and the trial was repeated after the standard 30-sec ITI. In other words, a correction procedure was used.

Following acquisition, the RI was increased between sessions to $1,2,4$, or $8 \mathrm{sec}$. On alternating sessions, the RI was returned to $0.5 \mathrm{sec}$, followed by the next $\mathrm{RI}$ on the following day (e.g., 0.5 , $1,0.5,2,0.5,4,0.5,8,0.5 \mathrm{sec}$, etc.). This sequence of RIs was repeated twice. During these sessions, the correction procedure was not in effect. The RI was then returned to $0.5 \mathrm{sec}$ and the correction procedure was reinstated for the next five sessions. In the final phase, the durations of the ITI and the RI were manipulated factorially over the 15 sessions. Each of these sessions contained one of three ITI durations (i.e., 5, 10, or $30 \mathrm{sec}$ ) and one of five RIs (i.e., $0.5,1,2,4$, or $8 \mathrm{sec}$ ). All possible ITI and RI combinations were presented in random order; the correction procedure was not in effect during this phase.

\section{RESULTS}

Figure 1 shows acquisition of matching (collapsed across short and long samples) for the temporal and visual groups. Acquisition of the DMTS task was more rapid for the visual than for the temporal group. A 2 (groups) $\times 32$ (sessions) mixed analysis of variance (ANOVA) performed on the acquisition data showed significant main effects for groups $[F(1,12)=20.12, p<.001]$ and sessions $[F(31,372)=45.95, p<.001]$, and a significant interaction between groups and sessions $[F(31,372)=$ $1.87, p<.004$ ]. Newman-Keuls post hoc tests conducted on the interaction revealed that visual matching was higher than temporal matching throughout acquisition; however, mean performance was not reliably different $(p>.05)$ during the last five sessions. In addition, analysis of individual performance during the first sessions failed to reveal any consistent bias for position or color. 


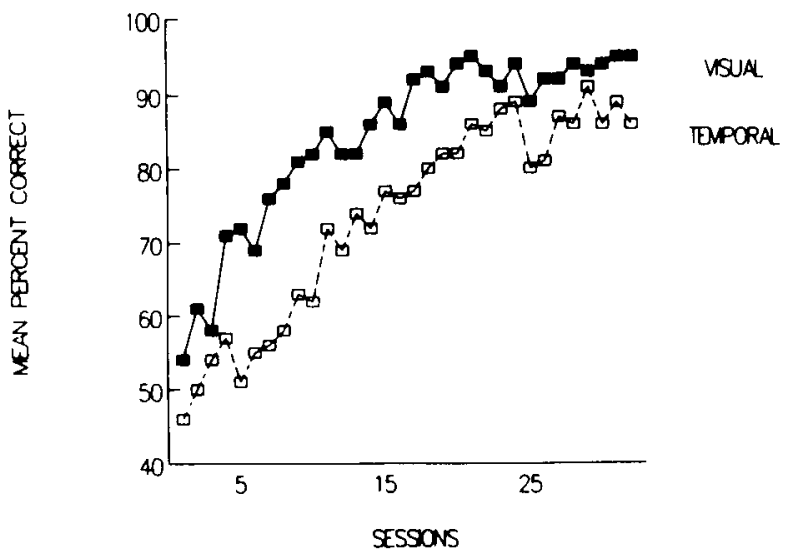

Figure 1. Acquisition of the delayed matching-to-sample task for the temporal and visual groups.

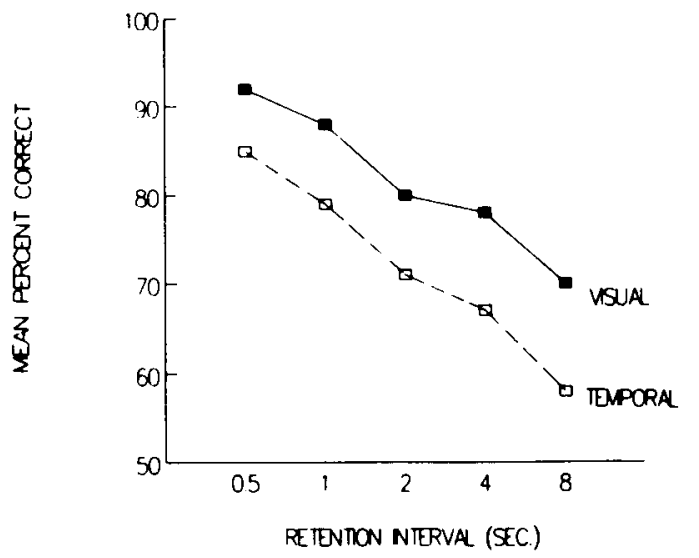

Figure 2. Mean percentage of correct matching for the visual and temporal groups across retention intervals, collapsed across sample duration.

Figure 2 shows mean accuracy collapsed across sample duration and color over the $0.5-8$-sec RIs when the ITI duration was held constant at $30 \mathrm{sec}$. Matching was higher in the visual group at each RI. More importantly, the slope of the retention curves was similar in each group, such that accuracy fell at a similar rate as the RI increased. When a mixed ANOVA was applied to the 2 (groups) $\times$ 2 (blocks of RI testing) $\times 2$ (sample durations) $\times 4$ (RIs) design, the analysis revealed significant main effects for groups $[F(1,12)=5.54, p<.05]$ and $\mathrm{RI}[F(4,48)=$ $43.55, p<.001]$. Although no interaction emerged between groups and RI, a significant interaction between groups, $\mathrm{RI}$, and sample duration was obtained $[F(4,48)$ $=3.10, p<.05]$. Figure 3 shows that this interaction was largely due to the differential effects produced by the short and long samples in each group. Graphically, the visual group showed higher performance with long samples during all but the 8-sec RI, where accuracy after short and long samples was equivalent. However, in the temporal group, short samples produced somewhat better matching with 0.5-2-sec RIs, but long samples produced better matching with 4- and 8-sec RIs. Newman-Keuls tests performed on the interaction showed that for the visual group, matching accuracy was greater after long than after short samples during the 1- and 4-sec RIs. In the temporal group, no reliable differences were shown following short and long samples. The analysis failed to show any reliable effects involving blocks of $\mathrm{RI}$ testing ( $p>.05$ in all cases).

Figure 4 shows mean performance across the 0.5-8sec RIs in the phase in which both the RI and the ITI durations were manipulated. Matching accuracy was higher in the visual group, but accuracy in both groups declined at a similar rate when the RI was increased. However, in contrast with the first phase of testing, Figure 5 shows that when the RI and the ITI were varied together, short and long samples produced reliably different performance in each group. In the visual group, accuracy was higher with long samples, whereas in the temporal group, accuracy was higher with short samples. A mixed ANOVA was performed on the 2 (groups) $\times 5$ (RIs) $\times 3$ (ITIs) $\times 2$ (sample durations) design and revealed significant main effects for groups $[F(1,12)=19.70, p<.01]$ and

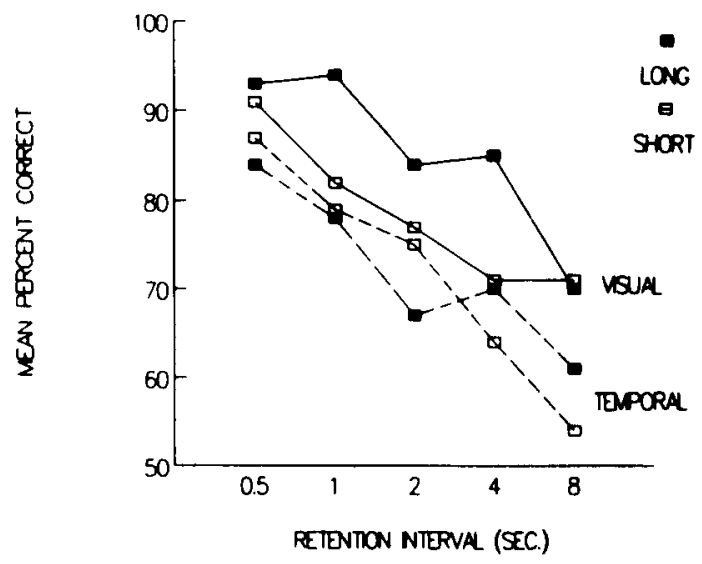

Figure 3. Mean percentage of correct matching for the temporal (dashed lines) and visual (solid lines) groups wth 5-sec (open squares) and 30-sec (filled squares) samples.

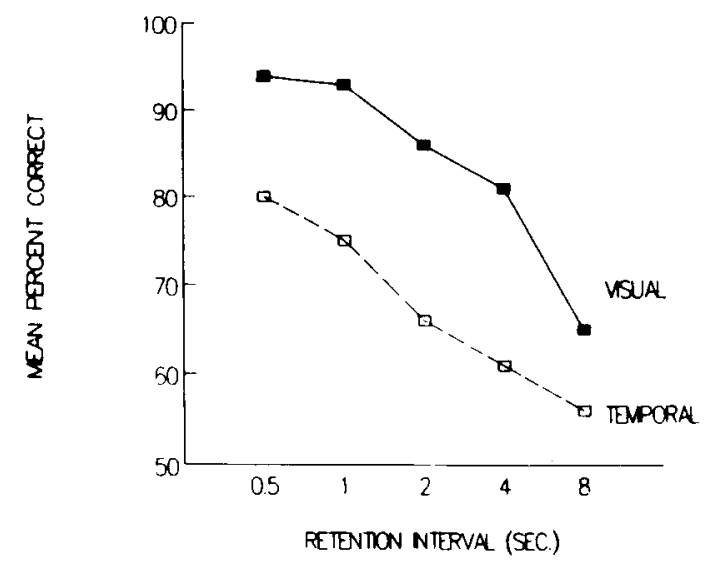

Figure 4. Visual and temporal matching performance acroes retention intervals in the phase in which the retention interval and the intertrial interval were varied together. 


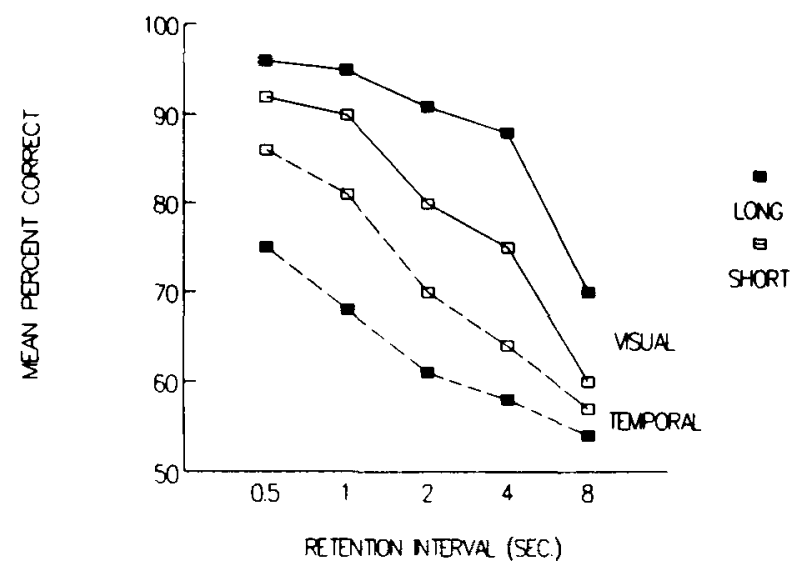

Figure 5. Visual (solid lines) and temporal (dashed lines) matching with 5-sec (open squares) and 30-sec (filled squares) samples, collapsed across intertrial interval.

$\mathrm{RI}[F(4,48)=62.03, p<.01]$. Although a significant interaction emerged between group and $\mathrm{RI}[F(4,48)=$ $2.83, p<.05$ ], Newman-Keuls tests revealed that visual matching was greater than temporal matching at all RIs $(p<.05)$.

The differential effects produced by sample duration were shown to be reliable in this phase, as indicated by the significant interaction between group and sample duration $[F(1,12)=16.40, p<.01]$. Newman-Keuls tests showed that the subjects in the visual group were more accurate on long-sample trials, whereas the subjects in the temporal group were more accurate on short-sample trials.

Finally, the analysis showed a significant main effect for ITI $[F(2,24)=3.43, p<.05]$. Although post hoc tests showed that both groups matched more accurately with the 30-sec ITI than with the 5-sec ITI (collapsed across groups), a significant interaction occurred between ITI and RI $[F(8,96)=3.20, p<.01]$. This interaction is illustrated in Figure 6. The figure shows performance in both groups with 5-, 10-, and 30-sec ITIs as a function

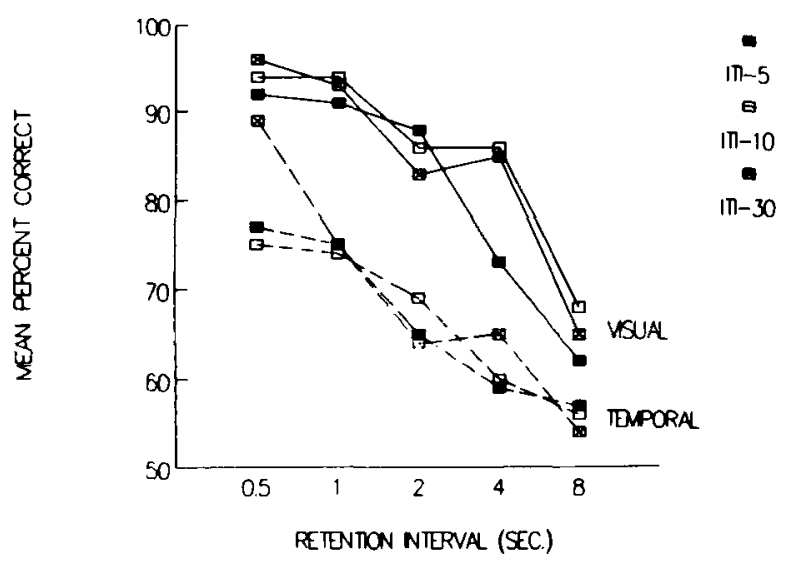

Figure 6. Mean performance for the visual (solid lines) and temporal (dashed lines) groups with the 5-, 10-, and 30-sec intertrial intervals, as a function of retention interval. of the $0.5-8-\mathrm{sec}$ RIs (collapsed across sample duration). Although each group seemed to match better with the 10and 30-sec ITIs, post hoc tests showed that the ITI effect was limited to the 0.5 - and 4-sec RIs. No interaction was found between group, RI, and ITI $(p>.05)$.

\section{DISCUSSION}

The purpose of the present experiment was to compare directly pigeons' STM of temporal and visual stimuli during a DMTS task. The results showed that acquisition of the DMTS task was more rapid for the visual than for the temporal group. Because both groups received identical sample and comparison stimuli, differing only in terms of the relevant dimension, the different rates of learning suggest that the temporal samples were more difficult to process than the visual samples. Indeed, visual matching was also superior to temporal matching when the RI and ITI variables were manipulated. However, although accuracy was higher in the visual than in the temporal group, accuracy at the end of acquisition was not reliably different, and rates of forgetting both samples were similar across the RI and ITI manipulations.

Recently, much DMTS research has been conducted to determine the content of animal STM (see, e.g., Honig \& Thompson, 1982; Roitblat, 1980; Stonebraker, 1981; Wasserman, 1986). According to a retrospective model of processing, animals preserve a memorial representation of the sample attributes throughout the RI. If the temporal and visual subjects in the present study processed the samples retrospectively, we would expect to find quantitative and/or qualitative differences in retention of these stimuli. For example, since the temporal DMTS task appeared to be more difficult, we might expect that the slope of the retention curves would be steeper in the temporal than in the visual group. A prospective model of STM, on the other hand, holds that samples activate instructional codes stored in long-term memory regarding the to-bechosen comparison. According to this view, the contents of STM would be similar for both groups, since each group was trained with the same color comparisons. The findings reported here generally support a prospective model, in that accuracy of both groups was similarly affected by the RI and ITI variables. These results are consistent with those obtained by Kraemer and Roberts (1984). In their study, separate groups of pigeons were trained with auditory and visual samples, each associated with blue and yellow comparisons. Their results showed that accuracy was higher in the visual than in the auditory group. More importantly, the slopes of the retention curves were similar as the RI increased, and performance similarly improved with longer ITIs.

Although rates of forgetting were similar in the present study, one important difference between the groups was accuracy following short and long samples. In the visual group, matching was generally higher with the $30-\mathrm{sec}$ than with the 5-sec samples. Past research has shown that matching typically improves with increasing sample du- 
ration (see Grant, 1976; Maki \& Leith, 1973; Roberts \& Grant, 1974), but some research has shown that accuracy declines with samples exceeding $10 \mathrm{sec}$ (see Grant \& MacDonald, 1986; Santi, Grossi, \& Gibson, 1982). It has been suggested (Santi et al., 1982) that reduced accuracy shown with long samples is due to extinction processes thought to occur with prolonged sample exposure. Perhaps one reason why long samples led to higher matching in the present study was due to the procedure used. Specifically, in many studies, long samples are not presented consistently throughout training, but instead appear occasionally on probe trials. In contrast, in the present procedure, the samples were presented according to a fixed 5- or 30sec schedule, each occurring equally often in the presence of the red and green lights.

In the temporal group, short samples produced higher matching than did long samples in the condition in which the RI and the ITI were varied together (see Figure 5). The finding that temporal matching is more accurate after short than after long samples has been demonstrated previously (Kraemer et al., 1985; Spetch \& Wilkie, 1982, 1983). A unique finding, however, was that the retention curves were similar for short and long durations. Indeed, several studies (e.g., Spetch \& Wilkie, 1982, 1983) have shown that memory declines more rapidly with long samples. In Spetch and Wilkie's work, samples were durations of 2 and $10 \mathrm{sec}$, and the comparisons were blue and yellow fields. They found that at longer RI durations (e.g., 10-20 sec), subjects showed an increased tendency to choose the comparison associated with the short sample. Their results suggested that memory for event duration shortened over the RI, producing a choose-short bias. The subjective shortening model assumes that subjects preserve a representation of sample duration, a process consistent with a retrospective model of STM. An alternative account, incorporating prospective coding (Kraemer et al., 1985), is that short and long samples activate instructional codes regarding the to-be-chosen comparison. As the RI increases, pigeons eventually forget the response code and choose the comparison associated with the short sample because the absence of a response code is more similar to that associated with the code for a short than for a long sample. Thus, performance differences after short and long samples would be expected only at rather long RIs.

Although the present retention data are interpreted more readily by the prospective coding hypothesis, this view does not explain why accuracy was typically higher on short-sample trials in the temporal group. One possibility is that in the temporal group, long samples might initially activate a code associated with the short sample, producing reduced accuracy due to interference (for a related discussion, see Grant \& Roberts, 1973; Roberts \& Grant, 1976; Wright, Urcuioli, \& Sands, 1986; Zentall \& Hogan, 1974, 1977). Another explanation involves the procedure used here. Specifically, in the prior DMTS studies involving temporal samples, the samples were relatively short (e.g., 2 and $10 \mathrm{sec}$ ), and terminated automat- ically after the duration timed out. In the present procedure, however, sample termination was scheduled according to a fixed-interval (FI) 5- or 30-sec schedule. Thus, the samples were longer and they required a response to be terminated. Perhaps the FI-30 samples led to poorer performance due to decreased attention. Although virtually all pigeons responded reliably throughout the sample duration, it did appear that the rate of responding decreased when the sample duration exceeded $5 \mathrm{sec}$.

Another procedural difference was that the RIs employed here were brief $(1-8 \mathrm{sec})$ relative to those of prior research (i.e., 10-20 sec). Perhaps different retention functions would emerge if longer RIs were used. Whether the results were due to procedural variables or interference is unclear, and the issues raised here provide the focus of our current research.

\section{REFERENCES}

Cohen, S. L., Calisto, G., \&entz, B. E. (1981). Comparisons of sample stimuli in delayed symbolic matching-to-sample: Some results and implications. Psychological Record, 31, 77-93.

Cook, R. G. (1980). Retroactive interference in pigeon short-term memory by a reduction in ambient illumination. Joumal of Experimental Psychology: Animal Behavior Processes, 6, 326-338.

Grant, D. S. (1976). Effect of sample presentation time on long delay matching in the pigeon. Learning \& Motivation, 7, 580-590.

Grant, D. S., MacDonald, S. E. (1986). Matching to element and compound samples in pigeons: The role of sample coding. Journal of Experimental Psychology: Animal Behavior Processes, 12, 160-171.

Grant, D. S., \& RoBerTs, W. A. (1973). Trace interaction in pigeon short-term memory. Journal of Experimental Psychology, 101, $21-29$.

Herman, L. M., \& Forestell, P. H. (1985). Short-term memory in pigeons: Modality-specific or code-specific effects? Animal Leaming \& Behavior, 13, 463-465.

HoNIG, W. K. (1978). Studies of working memory in the pigeon. In S. H. Hulse, H. Fowler, \& W. K. Honig (Eds.), Cognitive processes in animal behavior (pp. 211-248). Hillsdale, NJ: Erlbaum.

HoniG, W. K., ThOMPSON, R. K. R. (1982). Retrospective and prospective processing in animal working memory. In G. H. Bower (Ed.), The psychology of learning and motivation (Vol. 16, pp. 239283). New York: Academic Press.

Kendrick, D. F., RILLING, M. (1984). AIM: A theory of active and inactive memory. In D. F. Kendrick, M. R. Rilling, \& M. R. Denny (Eds.), Theories of animal memory (pp. 129-152). Hillsdale, NJ: Erlbaum.

Kendrick, D. F., Rilling, M., Denny, M. R. (1986). Theories of animal memory. Hillsdale, NJ: Erlbaum.

Krazmer, P. J., Mazmanian, D. S., Roberts, W. A. (1985). The choose-short effect in pigeon memory for stimulus duration: Subjective shortening versus coding models. Animal Learning \& Behavior, 13, 349-354.

Kraemer, P. J., Roberts, W. A. (1984). Short-term memory for visual and auditory stimuli in pigeons. Animal Learning \& Behavior, 12, 275-284

MAKI, W. S., JR., Lerth, C. R. (1973). Shared attention in pigeons. Journal of the Experimental Analysis of Behavior, 19, 345-349.

Roberts, W. A., \& Grant, D. S. (1974). Short-term memory in the pigeon with presentation time precisely controlled. Leaming \& Motivation, 5, 393-408.

Roberts, W. A., \& Grant, D. S. (1976). Studies of short-term memory in the pigeon using the delayed matching to sample procedure. In D. L. Medin, W. A. Roberts, \& R. T. Davis (Eds.), Processes of animal memory (pp. 79-112). Hillsdale, NJ: Erlbaum.

Roitblat, H. L. (1980). Codes and coding processes in pigeon shortterm memory. Animal Learning \& Behavior, 8, 341-351. 
Roitblat, H. L., Bever, T. G., \& Terrace, H. S. (1984). Animal cognition. Hillsdale, $\mathrm{NJ}$ : Erlbaum.

Santi, A., Grossi, V., \& Gibson, M. (1982). Differences in matchingto-sample performance with element and compound sample stimuli in pigeons. Learning \& Motivation, 13, 240-256.

SPETCH, M. A., \& WILKIE, D. M. (1982). A systematic bias in pigeons' memory for food and light durations. Behaviour Analysis Letters, 2, 267-274.

SPETCH, M. A., \& WiLkiE, D. M. (1983). Subjective shortening: A model of pigeons' memory for event duration. Joumal of Experimental Psychology: Animal Behavior Processes, 9, 14-30.

STONEBRAKER, T. B. (1981). Retrospective versus prospective processes in delayed matching-to-sample. Unpublished doctoral dissertation, Michigan State University, East Lansing, MI.

Wasserman, E. A. (1986). Prospection and retrospection as processes of animal short-term memory. In D. F. Kendrick, M. E. Rilling, \& M. R. Denny (Eds.), Theories of animal memory (pp. 53-75). Hillsdale, NJ: Erlbaum.

Wright, A. A., Urcuiol, P. J., SAnds, S. F. (1986). Proactive interference in animal memory. In D. F. Kendrick, M. E. Rilling, \& M. R. Denny (Eds.), Theories of animal memory (pp. 101-125). Hillsdale, NJ: Erlbaum.

Zentall, T. R., Hogan, D. E. (1974). Memory in the pigeon: Proactive inhibition in a delayed matching task. Bulletin of the Psychonomic Society, 4, 109-112.

Zentall, T. R., Hogan, D. E. (1977). Short-term proactive inhibition in the pigeon. Leaming \& Motivation, 8, 367-386.

(Manuscript received October 15, 1988; revision accepted for publication July 19, 1989.) 